Supporting Information

for

\title{
Amido Pincer Complexes of Palladium: Synthesis, Structure, and Catalytic Heck Reaction
}

Lan-Chang Liang and Mei-Hui Huang

\section{Contents}

1. Figure S1. The Hammett plot for the competitive reactions of aryl bromides with styrene

2. X-ray crystallographic report of [PNP]PdCl

\section{The Hammett plot}

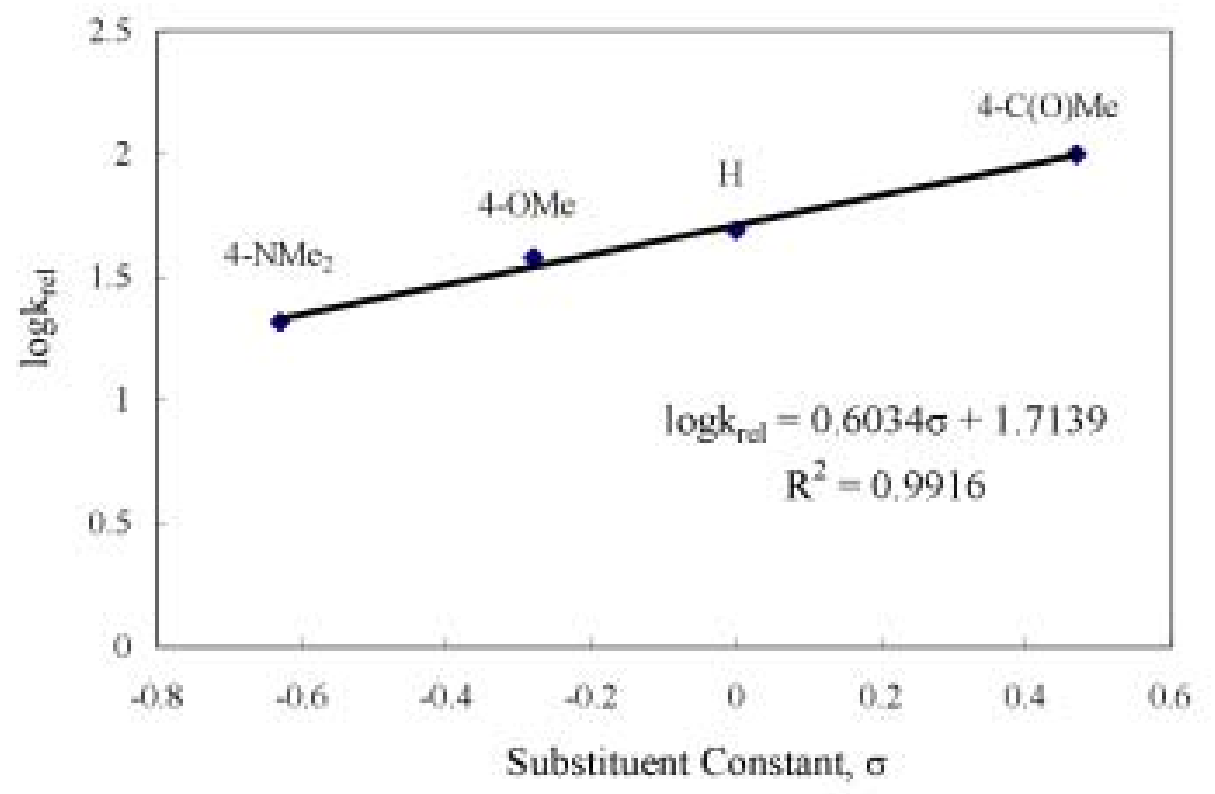

Figure S1. Hammett plot for the coupling of aryl bromides (92 mM) with styrene catalyzed by

3a $(0.92 \mathrm{mM})$ in NMP at $160{ }^{\circ} \mathrm{C}$, providing the reaction constant $\rho=0.6034$. 


\section{X-ray crystallographic report of [PNP]PdCl}

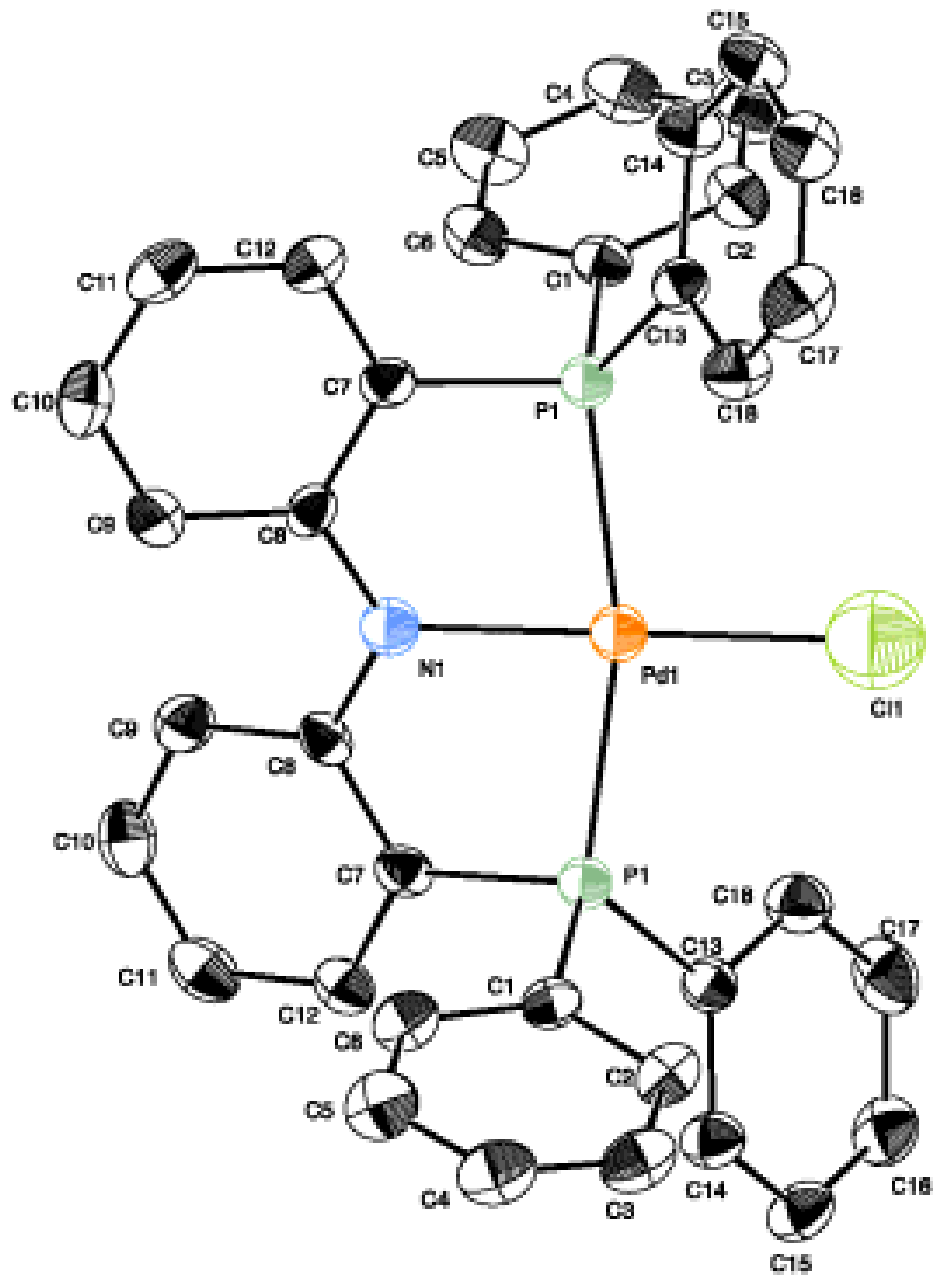




\section{Abstract \\ We present the crystal and molecular structure of [PNP]PdCl.}

\section{Comment}

The study of the titled structure was undertaken to establish its three dimensional structure. Geometries are tabulated below. All diagrams and calculations were performed using maXus (Bruker Nonius, Delft \& MacScience, Japan).

Size: $0.12 \times 0.10 \times 0.07$

\section{Experimental}

Crystal data

$$
\begin{aligned}
& \mathrm{C}_{36} \mathrm{H}_{28} \mathrm{ClNP}_{2} \mathrm{Pd} \\
& \mathrm{C}_{36} \mathrm{H}_{28} \mathrm{ClNP}_{2} \mathrm{Pd} \\
& \mathrm{M}_{\mathrm{r}}=678.428 \\
& \text { Orthorhombic } \\
& \text { Fdd2 } \\
& \mathrm{a}=18.5222(8) \AA \\
& \mathrm{b}=32.877(2) \AA \\
& \mathrm{c}=9.9178(4) \AA \\
& \alpha=90.00^{\circ} \\
& \beta=90.00^{\circ} \\
& \gamma=90.00^{\circ} \\
& \mathrm{V}=6039.5(5) \AA^{3} \\
& \mathrm{Z}=8 \\
& D_{\mathrm{x}}=1.492 \mathrm{Mg} \mathrm{m}^{-3}
\end{aligned}
$$

Density measured by: not measured fine-focus sealed tube

Mo $K \alpha$ radiation

$\lambda=0.71073$

Cell parameters from 7804

$\theta=2.037-25.028^{\circ}$

$\mu=0.836 \mathrm{~mm}^{-1}$

$\mathrm{T}=298 \mathrm{~K}$

Cube

black

Crystal source:Local laboratory

\section{Data collection}

\section{KappaCCD}

CCD

Absorption correction: multi-scan

$\mathrm{T}_{\min }=0.860, \mathrm{~T}_{\max }=0.923$

5939 measured reflections

2399 independent reflections

2115 observed reflections

Criterion: >2sigma(I)

$\theta_{\max }=24.98^{\circ}$

$\mathrm{h}=-22 \rightarrow 18$

$\mathrm{k}=-31 \rightarrow 38$

$1=-11 \rightarrow 9$

Refinement

Refinement on $F^{2}$ fullmatrix least squares refinement

$\mathrm{R}(\mathrm{all})=0.0583$

$\mathrm{R}(\mathrm{gt})=0.0410$

$w R($ ref $)=0.1531$

$w R(g t)=0.1099$

$\mathrm{S}(\mathrm{ref})=1.117$

2399 reflections

182 parameters

1 restraints

Only coordinates of $\mathrm{H}$ atoms refined

Calculated weights calc

$\Delta / \sigma_{\max }=0.001$

$\Delta \rho_{\max }=0.587 \mathrm{e}^{3}$

$\Delta \rho_{\min }=-1.237 \mathrm{e}^{3}$

Extinction correction: none

Atomic scattering factors from International

Tables Vol C Tables 4.2.6.8 and 6.1.1.4

Flack parameter $=-0.02(6)$

Flack H D (1983), Acta Cryst. A39, 876-881

Data collection: KappaCCD

Cell refinement: HKL Scalepack (Otwinowski \& Minor 1997)

Data reduction: Denzo and Scalepak (Otwinowski \& Minor, 1997)

Program(s) used to solve structure: SIR92

(Altomare et al., 1994)

Program(s) used to refine structure: SHELXL-97

(Sheldrick, 1997) 
Table . Fractional atomic coordinates and equivalent isotropic thermal parameters $\left(\AA^{2}\right)$

$\begin{array}{llllll} & \mathrm{x} & \mathrm{y} & \mathrm{z} & \mathrm{U}_{\text {eq }} & \text { Occ } \\ \text { Pd1 } & 0.0000 & 0.0000 & 0.83279(7) & 0.0401(3) & 1 \\ \text { C11 } & 0.0000 & 0.0000 & 1.0660(5) & 0.0823(16) & 1 \\ \text { P1 } & 0.07221(9) & 0.05624(6) & 0.8030(2) & 0.0400(5) & 1 \\ \text { N1 } & 0.0000 & 0.0000 & 0.6255(11) & 0.038(3) & 1 \\ \text { C1 } & 0.1692(4) & 0.0464(2) & 0.7946(9) & 0.0419(17) & 1 \\ \text { C2 } & 0.2126(5) & 0.0475(3) & 0.9091(11) & 0.057(2) & 1 \\ \text { C3 } & 0.2856(5) & 0.0382(3) & 0.8996(12) & 0.063(3) & 1 \\ \text { C4 } & 0.3142(5) & 0.0277(3) & 0.7795(12) & 0.066(3) & 1 \\ \text { C5 } & 0.2731(5) & 0.0268(3) & 0.6668(12) & 0.066(3) & 1 \\ \text { C6 } & 0.2004(4) & 0.0366(3) & 0.6717(10) & 0.051(2) & 1 \\ \text { C7 } & 0.0477(3) & 0.0682(2) & 0.6309(8) & 0.0411(18) & 1 \\ \text { C8 } & 0.0137(4) & 0.0369(2) & 0.5606(8) & 0.0395(18) & 1 \\ \text { C9 } & -0.0077(4) & 0.0452(3) & 0.4260(10) & 0.054(2) & 1 \\ \text { C10 } & 0.0072(5) & 0.0824(3) & 0.3681(9) & 0.062(3) & 1 \\ \text { C11 } & 0.0431(5) & 0.1121(3) & 0.4378(11) & 0.061(2) & 1 \\ \text { C12 } & 0.0624(4) & 0.1055(2) & 0.5704(9) & 0.0474(18) & 1 \\ \text { C13 } & 0.0604(4) & 0.1022(2) & 0.9022(8) & 0.0440(18) & 1 \\ \text { C14 } & 0.1126(4) & 0.1326(3) & 0.9080(9) & 0.052(2) & 1 \\ \text { C15 } & 0.0993(5) & 0.1677(3) & 0.9765(10) & 0.059(2) & 1 \\ \text { C16 } & 0.0340(5) & 0.1740(3) & 1.0384(10) & 0.065(2) & 1 \\ \text { C17 } & -0.0188(6) & 0.1439(3) & 1.0353(11) & 0.072(3) & 1 \\ \text { C18 } & -0.0053(5) & 0.1084(3) & 0.9681(11) & 0.058(2) & 1 \\ \text { H2 } & 0.1913 & 0.0551 & 0.9937 & 0.069 & 1 \\ \text { H3 } & 0.3163 & 0.0371 & 0.9774 & 0.075 & 1 \\ \text { H4 } & 0.3647 & 0.0218 & 0.7701 & 0.079 & 1 \\ \text { H5 } & 0.2945 & 0.0202 & 0.5814 & 0.079 & 1 \\ \text { H6 } & 0.1706 & 0.0359 & 0.5925 & 0.062 & 1 \\ \text { H9 } & -0.0329 & 0.0240 & 0.3788 & 0.065 & 1 \\ \text { H10 } & -0.0070 & 0.0879 & 0.2768 & 0.074 & 1 \\ \text { H11 } & 0.0553 & 0.1374 & 0.3955 & 0.073 & 1 \\ \text { H12 } & 0.0860 & 0.1265 & 0.6215 & 0.057 & 1 \\ \text { H14 } & 0.1581 & 0.1290 & 0.8632 & 0.063 & 1 \\ \text { H15 } & 0.1356 & 0.1885 & 0.9822 & 0.070 & 1 \\ \text { H16 } & 0.0233 & 0.1988 & 1.0854 & 0.078 & 1 \\ \text { H17 } & -0.0638 & 0.1485 & 1.0807 & 0.087 & 1 \\ \text { H18 } & -0.0410 & 0.0873 & 0.9629 & 0.069 & 1\end{array}$

$$
U_{e q}=1 / 3 \quad{ }_{i} \quad{ }_{j} U_{i j} a_{i} * a_{j} * \boldsymbol{a}_{i} \cdot \boldsymbol{a}_{j} .
$$

$0.6309(8) \quad 0.0411(18) \quad 1$

$\begin{array}{lll}0.5606(8) & 0.0395(18) & 1 \\ 0.4260(10) & 0.054(2) & 1\end{array}$

$0.3681(9) \quad 0.062(3) \quad 1$

$\begin{array}{lll}0.5704(9) & 0.0474(18) & 1 \\ 0.9022(8) & 0.0440(18) & 1\end{array}$

Table . Anisotropic displacement parameters $\left(\AA^{2}\right)$

$\begin{array}{lllllll} & \mathrm{U}_{11} & \mathrm{U}_{12} & \mathrm{U}_{13} & \mathrm{U}_{22} & \mathrm{U}_{23} & \mathrm{U}_{33} \\ \text { Pd1 } & 0.0455(4) & -0.0059(4) & 0.000 & 0.0374(4) & 0.000 & 0.0375(4) \\ \text { C11 } & 0.138(5) & -0.0151(17) & 0.000 & 0.064(3) & 0.000 & 0.044(3) \\ \text { N1 } & 0.048(6) & -0.008(4) & 0.000 & 0.047(6) & 0.000 & 0.019(5) \\ \text { C1 } & 0.042(4) & 0.001(3) & -0.004(3) & 0.033(3) & -0.002(3) & 0.051(5) \\ \text { C2 } & 0.060(5) & 0.000(4) & -0.010(4) & 0.055(5) & -0.007(4) & 0.057(6) \\ \text { C3 } & 0.055(5) & 0.006(4) & -0.028(5) & 0.056(6) & 0.001(5) & 0.076(7) \\ \text { C4 } & 0.046(5) & 0.007(5) & -0.008(5) & 0.065(6) & -0.007(5) & 0.087(8) \\ \text { C5 } & 0.051(4) & 0.011(5) & 0.007(5) & 0.064(6) & -0.018(6) & 0.082(8) \\ \text { C6 } & 0.043(4) & 0.007(4) & -0.009(4) & 0.050(5) & -0.004(4) & 0.062(6) \\ \text { C7 } & 0.029(3) & -0.002(3) & -0.002(3) & 0.048(4) & 0.008(3) & 0.046(5) \\ \text { C8 } & 0.032(3) & 0.002(3) & -0.001(3) & 0.048(4) & 0.013(4) & 0.038(5) \\ \text { C9 } & 0.052(5) & -0.008(4) & -0.011(4) & 0.061(5) & 0.004(4) & 0.050(6) \\ \text { C10 } & 0.072(6) & 0.012(5) & -0.003(4) & 0.070(6) & 0.012(4) & 0.044(7) \\ \text { C11 } & 0.067(5) & 0.003(4) & 0.010(5) & 0.046(5) & 0.020(5) & 0.071(7)\end{array}$




$\begin{array}{lllllll}\mathrm{C} 12 & 0.047(4) & 0.002(3) & 0.008(4) & 0.042(4) & 0.005(4) & 0.053(5) \\ \text { C13 } & 0.047(4) & 0.000(3) & -0.001(3) & 0.041(4) & 0.002(3) & 0.044(5) \\ \text { C14 } & 0.051(4) & -0.003(4) & 0.002(4) & 0.044(5) & -0.005(4) & 0.061(6) \\ \text { C15 } & 0.064(5) & -0.003(4) & -0.003(4) & 0.046(5) & -0.017(4) & 0.065(6) \\ \text { C16 } & 0.087(6) & 0.004(5) & 0.006(5) & 0.044(5) & -0.013(5) & 0.064(7) \\ \text { C17 } & 0.076(5) & 0.013(5) & 0.029(5) & 0.070(7) & -0.009(6) & 0.071(7) \\ \text { C18 } & 0.057(5) & -0.004(4) & 0.006(4) & 0.050(5) & -0.002(5) & 0.066(7)\end{array}$

\begin{tabular}{|c|c|}
\hline Pd1-N1 & $2.056(11)$ \\
\hline $\mathrm{Pd} 1-\mathrm{P} 1^{\mathrm{i}}$ & $2.3009(18)$ \\
\hline Pd1-P1 & $2.3010(18)$ \\
\hline Pd1-Cl1 & $2.313(5)$ \\
\hline P1-C7 & $1.810(8)$ \\
\hline P1-C13 & $1.816(8)$ \\
\hline P1-C1 & $1.828(7)$ \\
\hline N1-C8 & $1.396(9)$ \\
\hline $\mathrm{N} 1-\mathrm{C}^{\mathrm{i}}$ & $1.396(9)$ \\
\hline C1-C6 & $1.387(12)$ \\
\hline $\mathrm{C} 1-\mathrm{C} 2$ & $1.392(12)$ \\
\hline $\mathrm{C} 2-\mathrm{C} 3$ & $1.389(14)$ \\
\hline $\mathrm{C} 3-\mathrm{C} 4$ & $1.349(16)$ \\
\hline C4-C5 & $1.353(14)$ \\
\hline C5-C6 & 1.385 (12) \\
\hline C7-C12 & $1.392(11)$ \\
\hline C7-C8 & $1.393(11)$ \\
\hline C8-C9 & $1.420(13)$ \\
\hline C9-C10 & $1.378(14)$ \\
\hline C10-C11 & $1.368(14)$ \\
\hline C11-C12 & $1.381(14)$ \\
\hline C13-C14 & $1.392(11)$ \\
\hline C13-C18 & $1.397(11)$ \\
\hline C14-C15 & $1.361(12)$ \\
\hline C15-C16 & $1.372(14)$ \\
\hline C16-C17 & $1.390(15)$ \\
\hline C17-C18 & 1.367 (14) \\
\hline $\mathrm{C} 2-\mathrm{H} 2$ & 0.9603 \\
\hline C3-H3 & 0.9592 \\
\hline C4-H4 & 0.9598 \\
\hline C5-H5 & 0.9600 \\
\hline C6-H6 & 0.9603 \\
\hline C9-H9 & 0.9606 \\
\hline C10-H10 & 0.9601 \\
\hline C11-H11 & 0.9586 \\
\hline C12-H12 & 0.9616 \\
\hline C14-H14 & 0.9600 \\
\hline C15-H15 & 0.9607 \\
\hline C16-H16 & 0.9599 \\
\hline C17-H17 & 0.9593 \\
\hline C18-H18 & 0.9597 \\
\hline $\mathrm{N} 1-\mathrm{Pd} 1-\mathrm{P} 1^{\mathrm{i}}$ & $82.63(6)$ \\
\hline N1-Pd1-P1 & $82.63(6)$ \\
\hline $\mathrm{P} 1{ }^{\mathrm{i}}-\mathrm{Pd} 1-\mathrm{P} 1$ & $165.27(11)$ \\
\hline N1-Pd1-Cl1 & $180.0000(10)$ \\
\hline $\mathrm{P} 1^{\mathrm{i}}-\mathrm{Pd} 1-\mathrm{C} 11$ & $97.37(6)$ \\
\hline P1-Pd1-Cl1 & $97.37(6)$ \\
\hline C7-P1-C13 & $107.4(4)$ \\
\hline C7-P1-C1 & $104.0(4)$ \\
\hline
\end{tabular}




\begin{tabular}{|c|c|}
\hline C13-P1-C1 & $106.9(3)$ \\
\hline C7-P1-Pd1 & $98.6(2)$ \\
\hline C13-P1-Pd1 & $122.0(3)$ \\
\hline C1-P1-Pd1 & $115.8(2)$ \\
\hline C8-N1-C8 ${ }^{\mathrm{i}}$ & $125.2(10)$ \\
\hline C8-N1-Pd1 & $117.4(5)$ \\
\hline C8 ${ }^{\mathrm{i}}-\mathrm{N} 1-\mathrm{Pd} 1$ & $117.4(5)$ \\
\hline C6-C1-C2 & $118.9(8)$ \\
\hline C6-C1-P1 & $119.4(6)$ \\
\hline C2-C1-P1 & $121.7(7)$ \\
\hline $\mathrm{C} 3-\mathrm{C} 2-\mathrm{C} 1$ & $120.1(9)$ \\
\hline $\mathrm{C} 4-\mathrm{C} 3-\mathrm{C} 2$ & $119.9(9)$ \\
\hline C3-C4-C5 & $121.0(8)$ \\
\hline C4-C5-C6 & $120.9(9)$ \\
\hline C5-C6-C1 & $119.3(8)$ \\
\hline C12-C7-C8 & $121.6(7)$ \\
\hline C12-C7-P1 & $123.3(6)$ \\
\hline C8-C7-P1 & $115.1(6)$ \\
\hline C7-C8-N1 & $119.6(7)$ \\
\hline C7-C8-C9 & $117.0(7)$ \\
\hline N1-C8-C9 & $123.3(8)$ \\
\hline C10-C9-C8 & $120.5(8)$ \\
\hline C11-C10-C9 & $121.3(8)$ \\
\hline C10-C11-C12 & $119.8(8)$ \\
\hline C11-C12-C7 & $119.8(8)$ \\
\hline C14-C13-C18 & $118.8(8)$ \\
\hline C14-C13-P1 & $122.4(6)$ \\
\hline C18-C13-P1 & $118.7(6)$ \\
\hline C15-C14-C13 & $120.2(8)$ \\
\hline C14-C15-C16 & 120.7 (9) \\
\hline C15-C16-C17 & $120.2(9)$ \\
\hline C18-C17-C16 & $119.4(9)$ \\
\hline C17-C18-C13 & 120.7 (9) \\
\hline C3-C2-H2 & 121.1 \\
\hline $\mathrm{C} 1-\mathrm{C} 2-\mathrm{H} 2$ & 118.8 \\
\hline C4-C3-H3 & 117.9 \\
\hline $\mathrm{C} 2-\mathrm{C} 3-\mathrm{H} 3$ & 122.0 \\
\hline C3-C4-H4 & 121.3 \\
\hline C5-C4-H4 & 117.6 \\
\hline C4-C5-H5 & 120.1 \\
\hline C6-C5-H5 & 119.0 \\
\hline C5-C6-H6 & 121.6 \\
\hline C1-C6-H6 & 119.0 \\
\hline С10-C9-H9 & 122.5 \\
\hline C8-C9-H9 & 117.0 \\
\hline C11-C10-H10 & 118.4 \\
\hline C9-C10-H10 & 120.4 \\
\hline C10-C11-H11 & 120.8 \\
\hline C12-C11-H11 & 119.4 \\
\hline C11-C12-H12 & 120.5 \\
\hline C7-C12-H12 & 119.6 \\
\hline C15-C14-H14 & 119.6 \\
\hline C13-C14-H14 & 120.1 \\
\hline C14-C15-H15 & 120.4 \\
\hline C16-C15-H15 & 118.9 \\
\hline C15-C16-H16 & 121.8 \\
\hline C17-C16-H16 & 118.0 \\
\hline C18-C17-H17 & 121.4 \\
\hline C16-C17-H17 & 119.2 \\
\hline C17-C18-H18 & 121.2 \\
\hline
\end{tabular}


Symmetry codes: (i) $\mathrm{x}, 1 / 2+\mathrm{y}, 1 / 2+\mathrm{z}$.

\section{References}

Mackay, S., Gilmore, C. J.,Edwards, C., Stewart, N. \& Shankland, K. (1999). maXus Computer Program for the Solution and Refinement of Crystal Structures. Bruker Nonius, The Netherlands, MacScience, Japan \& The University of Glasgow.

Johnson, C. K. (1976). ORTEP--II. A Fortran Thermal--Ellipsoid Plot Program. Report ORNL-5138. Oak Ridge National Laboratory, Oak Ridge, Tennessee, USA.

Otwinowski, Z. and Minor, W, (1997). In Methods in Enzymology, 276, edited by C. W. Carter, Jr. \& R. M. Sweet pp. 307--326, New York:Academic Press.

Altomare, A., Cascarano, G., Giacovazzo, C., Guagliardi, A., Burla, M. C., Polidori, G. \& Camalli, M. (1994). J. Appl. Cryst. 27, 435.

Sheldrick, G. M. (1997). SHELXL97. Program for the Refinement of Crystal Structures. University of Göttingen, Germany.

Blessing, R. H. (1995), Acta. Cryst. A51, 33--38. 